\title{
Polydopamine-induced hydroxyapatite coating facilitates hydroxyapatite/polyamide 66 implant osteogenesis: an in vitro and in vivo evaluation
}

This article was published in the following Dove Press journal: International Journal of Nanomedicine

\author{
Yanan $\mathrm{Xu}{ }^{\prime}$ \\ Hong $\mathrm{Li}^{2}$ \\ jieming $\mathrm{Wu}^{3}$ \\ Qiming Yang' \\ Dianming Jiang ${ }^{4}$ \\ Bo Qiao' \\ 'Department of Orthopedics, The \\ First Affiliated Hospital of Chongqing \\ Medical University, Chongqing, \\ People's Republic of China; ${ }^{2}$ Research \\ Center for Nano-Biomaterials, \\ Analytical and Testing Center, \\ Sichuan University, Chengdu, People's \\ Republic of China; ${ }^{3}$ Office of Teaching \\ Affairs, The First Affiliated Hospital \\ of Chongqing Medical University, \\ Chongqing, People's Republic of \\ China; ${ }^{4}$ Department of Orthopedics, \\ The Third Affiliated Hospital of \\ Chongqing Medical University, \\ Chongqing, People's Republic of China
}

Correspondence: Bo Qiao

Department of Orthopedics, The First Affiliated Hospital of Chongqing Medical University, No I Youyi Road, Yuzhong District, Chongqing 400016, People's Republic of China

Tel +862389011202

Fax $+86238901 \quad 1217$

Email qiaobo1985@I63.com

Dianming Jiang

Department of Orthopedics, The Third Affiliated Hospital of Chongqing Medical University, No I Shuanghu Road, Yubei District, Chongqing 40I I20, People's

Republic of China

Tel +86236035334l

Emailjdm571026@vip.163.com
Background: Hydroxyapatite/polyamide 66 (HA/P66) has been clinically used for several years owing to its good biocompatibility and bioactivity. However, it has been found that the osseointegration process of the HA/P66 implant takes a large amount of time because of the small amount of HA on its surface.

Methods: To increase the amount of HA and aid faster osseointegration, we prepared a HA coating using a biomimetic process assisted by polydopamine (PDA) on the HA/P66 substrate. The surface properties of the substrate modified by PDA and HA were characterized, and the capacity of biomaterials for osteogenic induction was investigated both in vitro and in vivo.

Results: The HA coating was successfully prepared on the HA/P66 substrate and verified by X-ray photoelectron spectroscopy (XPS), scanning electron microscopy (SEM), and X-ray diffraction (XRD). The HA coating remained firmly attached to the underlying PDA-HA/P66 substrate even after strong ultrasound treatment for $1 \mathrm{~h}$, and the calcium and phosphorus of the HA coating was continuously released in vitro in a slow manner. The formation of the HA coating on the PDA film greatly increased the hydrophilicity and surface roughness of HA/P66. In cell-based experiments, as compared with the HA/P66 substrate, the HA coating formation on the PDA film could facilitate the functions of $\mathrm{C} 3 \mathrm{H} 10 \mathrm{~T} 1 / 2$ cells, including cell adhesion, proliferation, spreading, alkaline phosphatase activity, calcium nodule formation, and expression of osteogenic differentiationrelated proteins. In addition, the HA/P66 scaffolds modified with PDA and HA coatings were implanted in rabbit femoral condyles. At 8 weeks after surgery, micro-computed tomography scanning (micro-CT) and hematoxylin-eosin (HE) staining revealed that more new bones were formed around the HA/P66 scaffold that was modified with a PDA-assisted HA coating.

Conclusion: These results indicate that the preparation of a PDA-assisted HA coating by using a biomimetic process significantly improves the capacity of biomaterials for osteogenic induction.

Keywords: hydroxyapatite, polydopamine, hydroxyapatite/polyamide 66, surface modification, osteogenic induction

\section{Introduction}

With the rapid development of medical technology, patients not only request the replacing or restoring of function of damaged and diseased tissues but also expect faster recuperation. ${ }^{1}$ The concept of orthopedic rapid rehabilitation has been accepted by an increasing number of patients. Rapid rehabilitation not only sets higher expectations for orthopedic surgeons but also presents a greater demand for bone implantation materials. Improving the capacity of biomaterials for osteogenic induction can achieve faster osseointegration, enable earlier implant loading, and shorten the treatment period. ${ }^{2}$ These results would not only allow rapid rehabilitation in patients but also decrease their 
health care costs. ${ }^{3}$ Therefore, more and more researchers have focused on the study of bone-implant interfaces. Hydroxyapatite/polyamide 66 (HA/P66) biomaterials have been clinically used for decades. Our group has carried out considerable basic research and clinical studies in this field. ${ }^{4-8} \mathrm{We}$ found that the amount of HA particles deposited on the surface of HA/P66 was relatively low, which affected the rate and quality of the initial osseointegration in HA/P66 implants. Different methods were employed to modify HA/P66 implants to promote osseointegration. Nell-1 gene-modified mesenchymal stem cells $^{9}$ and vascular endothelial growth factor ${ }^{6}$ were applied in combination with HA/P66. However, these methods were used only in the experimental stage and were difficult to apply clinically. Hence, we focused on finding a simple and effective method that could improve the capacity of HA/P66 for osteogenic induction and achieve faster osseointegration, thereby allowing easy application in the clinic.

Surface modification is an important method to improve the capacity of biomaterials for osteogenic induction. ${ }^{10}$ The preparation of HA coating is one of the most important methods in surface modification techniques. Thus far, there have been many techniques to produce HA coating on implant surfaces, such as plasma spraying, ${ }^{11}$ sol-gel techniques, ${ }^{12}$ and electrophoretic deposition. ${ }^{13}$ Unfortunately, these approaches usually require multiple steps, high temperature, special apparatus, and rigorous conditions that impede their application in the clinic. To avoid these limitations, researchers focused on a biomimetic method to synthesize the HA coating. ${ }^{14}$ The presence of a polydopamine (PDA) film provided an innovative biomimetic method for preparing the HA coating.

The PDA film was first produced by Messersmith who found that dopamine can produce a polymerized layer on almost any shape and material type by oxidative self-polymerization under alkaline conditions. ${ }^{15}$ Many studies showed that the PDA film was a simple and effective way to produce an HA coating on an implant surface. ${ }^{16,17}$ Currently, the most common PDA film method used for HA biomineralization is the PDA-assisted HA formation, a two-step approach. ${ }^{16}$ The PDA film is first prepared on the biomaterials and then reacted with $\mathrm{Ca}-\mathrm{P}$ ions in a simulated body fluid (SBF) solution to form the HA coating. In this study, we systematically studied the ability of the PDA-assisted HA coating to induce osteogenesis both in vitro and in vivo. We first prepared the HA coating on HA/P66 using a PDA-assisted biomimetic process. Then, surface characteristics such as chemistry, morphology, hydrophilicity, roughness, adhesion stability, and release behavior were studied. Finally, the capacity of the modified HA/P66 substrates for osteogenic induction was studied in vitro and in vivo.

\section{Materials and methods Preparation of PDA coating (denoted as PDA-HA/P66)}

The PDA coating was prepared according to a previously described method. ${ }^{15}$ Three types of HA/P66 substrates were used in this study - two $(\Phi 10 \mathrm{~mm}$ and $28 \times 20 \times 2 \mathrm{~mm})$ of which were used for in vitro experiments and one $(12 \times 4 \times 2 \mathrm{~mm})$ for animal experiments. The HA/P66 composite was provided by the Sichuan National Nanotechnology Co, Ltd (Chengdu, People's Republic of China) and had an HA content of approximately $40 \%$. All the substrates were produced by computer numerical control lathe (G-CNC300J; Guangzhou Machine Tool Works, Guangzhou, People's Republic of China). Prior to preparing the PDA film, the HA/P66 substrates were sonicated in deionized water for 30 minutes to clean the surfaces. Then, the dopamine solution ( $2 \mathrm{mg} / \mathrm{mL}$ ) was prepared by dissolving dopamine hydrochloride (H8502; Sigma-Aldrich Co., St Louis, MO, USA) in $10 \mathrm{mM}$ Tris buffer, and the $\mathrm{pH}$ was adjusted to 8.5 with an appropriate amount of $1 \mathrm{M} \mathrm{NaOH}$. Next, the substrates and dopamine solution were added into a beaker, and the exposed surface of the substrate to volume of the dopamine solution ratio was $10 \mathrm{~cm}^{2} / \mathrm{mL}$. Finally, the beaker was incubated for 24 hours in a $37^{\circ} \mathrm{C}$ shaker at constant temperature, and each substrate was thoroughly rinsed with deionized water and air-dried at room temperature for further use.

\section{PDA-assisted self-assembly of HA in SBF (denoted as HA-PDA-HA/P66)}

The deposition of HA on the PDA film was carried out in 1.5SBF following the method described previously. ${ }^{17}$ The PDA-HA/P66 substrates and 1.5SBF solution (Qingdao Jieshikang Biotechnology Co., Ltd, Shandong, People's Republic of China) were added into a beaker, and the exposed surface of the substrate to volume of the 1.5SBF solution ratio was $10 \mathrm{~cm}^{2} / \mathrm{mL}$. Then, the beaker was incubated in a $37^{\circ} \mathrm{C}$ shaker at constant temperature for 3 days. To provide adequate ions for HA formation, the SBF solution was refreshed every day. Finally, the substrates were rinsed with sufficient deionized water and air-dried at room temperature. All samples were disinfected with ethylene oxide before use.

\section{Material surface characterization}

The surface morphologies of HA/P66, PDA-HA/P66, and HA-PDA-HA/P66 were observed using a scanning electron microscopy (SEM; JSM-7500; JEOL, Tokyo, Japan). X-ray photoelectron spectroscopy (XPS; PHI 1800; ULVAC-PHI, Kanagawa, Japan) and X-ray diffraction (XRD; X'Pert proMPD; Philips, Eindhoven, the Netherlands) were employed 
to confirm the presence of the PDA and HA coating on the surface of HA/P66. The static water contact angle was measured with the sessile drop method using a Drop Shape Analyzer (DSA25; Krüss, Hamburg, Germany) at room temperature. Each sample was measured five times in different areas. The surface average roughness (Ra) was measured by a handheld roughness meter (TIME 3221; Beijing Time High Technology Ltd, Beijing, People's Republic of China). Ultrasonication tests were performed according to Ryu et al's ${ }^{17}$ method to compare the adhesion stability of the HA coating. The sample was ultrasonically treated $(35 \mathrm{kHz}$, $160 \mathrm{~W}$ ) in deionized water for 60 minutes and dried under vacuum for characterization. The weight of each sample was recorded before and after sonication. Then, the samples were examined by SEM to observe the difference in HA coating peeling off from the scaffold surface. The study of the release of calcium $(\mathrm{Ca})$ and phosphate $\left(\mathrm{PO}_{4}^{3-}\right)$ ions from the HA coating was performed in normal saline. The samples were placed in a polypropylene vial containing $10 \mathrm{~mL}$ of normal saline (five samples in each group, $\Phi 10 \mathrm{~mm}$ ) and incubated in a $37^{\circ} \mathrm{C}$ thermostat water bath for 7 days. The supernatant was collected $(5 \mathrm{~mL})$ after 1,4 , and 7 days, and the same volume of fresh normal saline was added to the polypropylene vial. The concentration of $\mathrm{Ca}$ and $\mathrm{PO}_{4}^{3-}$ ions in the collected supernatant was analyzed by inductively coupled plasma optical emission spectrometer (ICP-OES; iCAP 6300; Thermo Fisher Scientific, Waltham, MA, USA).

\section{Cell culture}

C3H10T1/2 cells, which are mouse mesenchymal stem cells derived from the mouse embryo, were used in this study (Cell Bank of Chinese Academy of Sciences, Shanghai, People's Republic of China). C3H10T1/2 cells were cultured in DMEM/F12 containing 10\% FBS and placed in a humidified atmosphere of $5 \% \mathrm{CO}_{2}$ at $37^{\circ} \mathrm{C}$. The culture medium was replaced every 3 days.

\section{Cell attachment and proliferation}

To determine the attachment of the $\mathrm{C} 3 \mathrm{H} 10 \mathrm{~T} 1 / 2$ cells on the material surfaces, the numbers of attached cells on the material surfaces after cocultivation with different samples for 6 and 12 hours were analyzed using a cell counting kit-8 (CCK-8) assay (Dojindo Molecular Technologies Inc., Kumamoto, Japan). First, three samples from each group were placed into a 24-well plate (Nest Biotechnology, Wuxi, People's Republic of China). Then, $5 \times 10^{4}$ viable cells were seeded into each well, and the culture plates were incubated in a humidified atmosphere of $5 \% \mathrm{CO}_{2}$ at $37^{\circ} \mathrm{C}$. At the scheduled time, PBS was used to rinse the samples for washing away the unattached cells, and the samples were then transferred to a new 24-well plate. Next, $50 \mu \mathrm{L}$ CCK-8 solution and $500 \mu \mathrm{L}$ DMEM/F12 containing 10\% FBS were added to each well, and the plates were incubated at $37^{\circ} \mathrm{C}$ in an incubator for 3 hours. After incubation, $100 \mu \mathrm{L}$ of the supernatant was taken from each well and added into a 96-well plate. The plate was then read at $450 \mathrm{~nm}$ with a Thermo Scientific microplate reader (Thermo Fisher Scientific).

The proliferation of the $\mathrm{C} 3 \mathrm{H} 10 \mathrm{~T} 1 / 2$ cells on the surface of the samples was also detected with the CCK- 8 assay. The protocol was similar to that mentioned earlier except that the cells were seeded in each well at a density of $2 \times 10^{4}$ and the incubating time points were 1, 4, and 7 days. SEM was used to observe cell proliferation on the material surfaces directly after coculturing for 3 and 7 days.

\section{Cell spreading}

The cells were seeded in each well at a density of $2 \times 10^{4}$, cocultured for 24 hours, and examined by SEM and confocal laser scanning microscopy (CLSM) to observe cell spreading. SEM was employed to visualize the cytoskeleton. First, PBS was used to carefully rinse the samples three times. The samples were then soaked in $2.5 \%$ glutaraldehyde for 15 minutes at room temperature to fix the cells in the samples. Next, the cells were dehydrated in an increasing ethanol gradient $(10 \%, 30 \%, 50 \%, 70 \%, 90 \%, 100 \%$, and $100 \% ; 10$ minutes each). Finally, after air-drying and gold sputtering, all the samples were observed using SEM, with the SEM images being measured using ImageJ software to analyze cell spreading area.

CLSM was employed to detect the filamentous actin of the cytoskeleton of the $\mathrm{C} 3 \mathrm{H} 10 \mathrm{~T} 1 / 2$ cells on the samples. In brief, the samples were washed gently with PBS three times. Then, the cells on the samples were fixed for 15 minutes with $4 \%$ paraformaldehyde. Next, the cells were permeabilized with $0.2 \%$ Triton $\mathrm{X}-100$ for 10 minutes, rinsed with PBS again, and stained with rhodamine-phalloidin $\left(50 \mu \mathrm{g} \cdot \mathrm{mL}^{-1}\right.$; Sigma-Aldrich Co.) at room temperature for 30 minutes. Finally, the cells were again washed three times with PBS and observed with CLSM (LSM800; Carl Zeiss Meditec AG, Jena, Germany).

\section{Cell culture of inductive osteogenesis}

Different sized samples were placed into six-well (for $28 \times 20 \times 2 \mathrm{~mm}$ samples) or 24 -well (for $\Phi 10 \mathrm{~mm}$ samples) plates for Western blotting, ALP activity assay, and alizarin red staining. Then, the $\mathrm{C} 3 \mathrm{H} 10 \mathrm{~T} 1 / 2$ cells were seeded at a density of $5 \times 10^{4}$ cells/well for the six-well plates and $2 \times 10^{4}$ cells/well for the 24 -well plates. After 24 hours of 
cocultivation, the DMEM/F12 culture medium was replaced with osteogenic induction medium (Cyagen Biosciences Co., Ltd., Guangzhou, People's Republic of China); the latter was replaced every 3 days.

\section{ALP activity assay}

After 7, 10, and 14 days of osteogenic induction, ALP activity was measured with an ALP assay kit (A059-2; Nanjing Jiancheng Bioengineering Institute, Nanjing, People's Republic of China) which follows the measurement principle of quantifying the amount of red perylenequinone derivatives. The cells were lysed with $1 \%$ Triton X-100 for 60 minutes to prepare the cell lysates. The quantification of ALP in the cell lysates was determined with the Thermo Scientific microplate reader at $520 \mathrm{~nm}$, and the protein concentration of cell lysates was measured with a Bicinchoninic Acid (BCA) protein assay kit (Beyotime, Jiangsu, People's Republic of China) in accordance with the manufacturer's instructions. Finally, the ALP activity was calculated according to the provided formula.

\section{Alizarin red staining and quantitative analysis}

Alizarin red staining was used to detect the formation of calcium nodules. After osteogenic induction for 21 days, the samples were harvested for alizarin red staining. The cells on the samples were washed gently with PBS three times and fixed with $4 \%$ paraformaldehyde for 15 minutes. Then, the samples were again washed with PBS three times and stained with $1 \%$ alizarin red solution (Cyagen Biosciences Co., Ltd.) for 45 minutes at room temperature. Next, the samples were rinsed adequately with PBS until the scrubbing solution became clear. The staining results were recorded using a digital camera. For quantitative analysis of alizarin red staining, the samples without cells were also stained with $1 \%$ alizarin red solution as per the abovementioned steps. Cetylpyridinium chloride (10\%; Sigma-Aldrich Co.) solution was used to dissolve the orange dye on the samples. The dissolving solution was harvested and measured with the Thermo Scientific microplate reader at $620 \mathrm{~nm}$.

\section{Western blot analysis}

Western blotting was used to detect indicators of osteogenic differentiation, such as collagen type I (COL 1), runt-related transcription factor 2 (RUNX2), and osteocalcin (OCN). After 14, 21, and 28 days of osteogenic induction, the cells on the samples were collected by trypsinization. The cells were lysed using RIPA lysis buffer (Beyotime) and centrifuged to obtain cell lysates. Protein quantification of whole cell lysates was performed using the BCA protein assay kit (Beyotime). Aliquots of cell lysates at a concentration of $20 \mu \mathrm{g} / \mu \mathrm{L}$ were loaded onto SDS-PAGE systems and transferred to polyvinylidene fluoride (PVDF) membranes. The membranes were then blocked with blocking solution (Beyotime) and incubated overnight with primary antibodies (1:1,000; Abcam, Cambridge, UK), followed by washing and incubations with anti-rabbit IgG secondary antibodies (Boster, Wuhan, People's Republic of China). The primary antibodies used were anti-COL1, anti-OCN, and anti-RUNX2; anti- $\beta$-actin was used for housekeeping. Finally, the Western blot membranes were exposed to Luminata Forte Western horseradish peroxidase (HRP) substrate (EMD Millipore, Billerica, MA, USA) and analyzed using a chemiluminescence system. The immunoblot bands were analyzed quantitatively with ImageJ software.

\section{Experimental animals and surgical procedure}

All animal experiment protocols were conducted according to the procedures approved by the Animal Care and Experiment Committee of The First Affiliated Hospital of Chongqing Medical University and approved by the animal ethics committee of The First Affiliated Hospital of Chongqing Medical University (no: 20187801). Nine adult, male New Zealand White rabbits weighing $2.1-2.8 \mathrm{~kg}$ were used in this study and randomly divided into three groups ( $\mathrm{n}=3$ each). Both femurs of each rabbit were used as surgical sites. Before surgery, general anesthesia was induced by intravenous injection $(1.0 \mathrm{~mL} / \mathrm{kg}$ ) using 3\% pentobarbital sodium solution (SigmaAldrich Co.). After anesthesia, the surgery area was shaved and disinfected with iodine. Lidocaine hydrochloride (2\%) was diluted with saline solution and injected subcutaneously at the surgical incision site to provide additional anesthesia. A longitudinal $3 \mathrm{~cm}$ lateral parapatellar incision was made, the patella was dislocated medially, and the femoral groove was exposed. With the knees flexed, the bone defects $(4 \times 2 \mathrm{~mm}$ and $12 \mathrm{~mm}$ depth) were created manually in the femoral groove with an osteotome (4 $\mathrm{mm}$ width and $2 \mathrm{~mm}$ thickness). Then, the HA/P66, PDA-HA/P66, and HA-PDA-HA/P66 implants were installed in the defects. The incisions were sutured in layers, and the lateral ligaments were sutured strongly to avoid patellar dislocation. Penicillin sodium (Southwest Pharmaceutical Co., Ltd, Chongqing, People's Republic of China; 200,000 IU/kg, every day, intramuscular injection) was administered for the first 3 days after surgery to reduce postoperative infection. The rabbits were kept in separate cages and allowed full activity after surgery. 


\section{Micro-computed tomography (micro-CT) detection}

Eight weeks after the surgery, nine rabbits were sacrificed by administering an overdose of sodium pentobarbital. Eighteen femoral condyles were harvested and immediately fixed in $4 \%$ paraformaldehyde for 72 hours. The samples were observed with a SCANCO microCT $\mu 100$ scanner (Scanco Medical, Brüttisellen, Switzerland; pixel size: $30 \mu \mathrm{m}$, scan voltage: $70 \mathrm{kV}$, scan current: $200 \mu \mathrm{A}$, integral time: $300 \mathrm{~ms}$ ). After scanning, the raw data were imported into the micro-CT software (Scanco Medical), the volume of interest (VOI) in three-dimensional (3D) images was reconstructed, and the trabecular number was calculated. The VOI was defined as a specific area within $1 \mathrm{~mm}$ from the implant surface. Finally, the percentage of bone volume (BV) to the total tissue volume (TV; BV/TV\%) within the VOI was calculated.

\section{Histological analysis}

After micro-CT scanning, the undecalcified samples were rinsed with distilled water and dehydrated stepwise for 3 days in an ascending series of ethanol (70\%-100\%) for histological examination. Then, the dehydrated samples were incubated in xylene for 3 days. Finally, all the samples were embedded in methyl methacrylate according to the manufacturer's instructions. The embedded samples were then longitudinally cut into $50 \mu \mathrm{m}$ sections using a diamond saw microtome (Leica SP-1600; Leica Microsystems, Wetzlar, Germany). H\&E staining was performed in the polymethylmethacrylate sections. The bone-implant contact (BIC) was measured using optical microscopy (Nikon Eclipse E200; Nikon Corporation, Tokyo, Japan). The percentage of BIC was determined using the following equation: $\mathrm{BIC}=$ bone contact length/implant length within one pitch $\times 100 \%$.

\section{Statistical analyses}

Quantitative data were presented as mean \pm SD. A two-way ANOVA was used for statistical analysis to determine any significant differences between different groups. Statistical analyses were performed with SPSS 18.0 (SPSS Inc., Chicago, IL, USA). $P<0.05$ was considered statistically significant.

\section{Results}

\section{Surface characterization and release behavior}

As shown in Figures 1 and 2, depositions of the PDA and HA coating on HA/P66 were verified by XPS and SEM. The XPS spectra of HA/P66, PDA-HA/P66, and HA-PDA-HA/P66 are

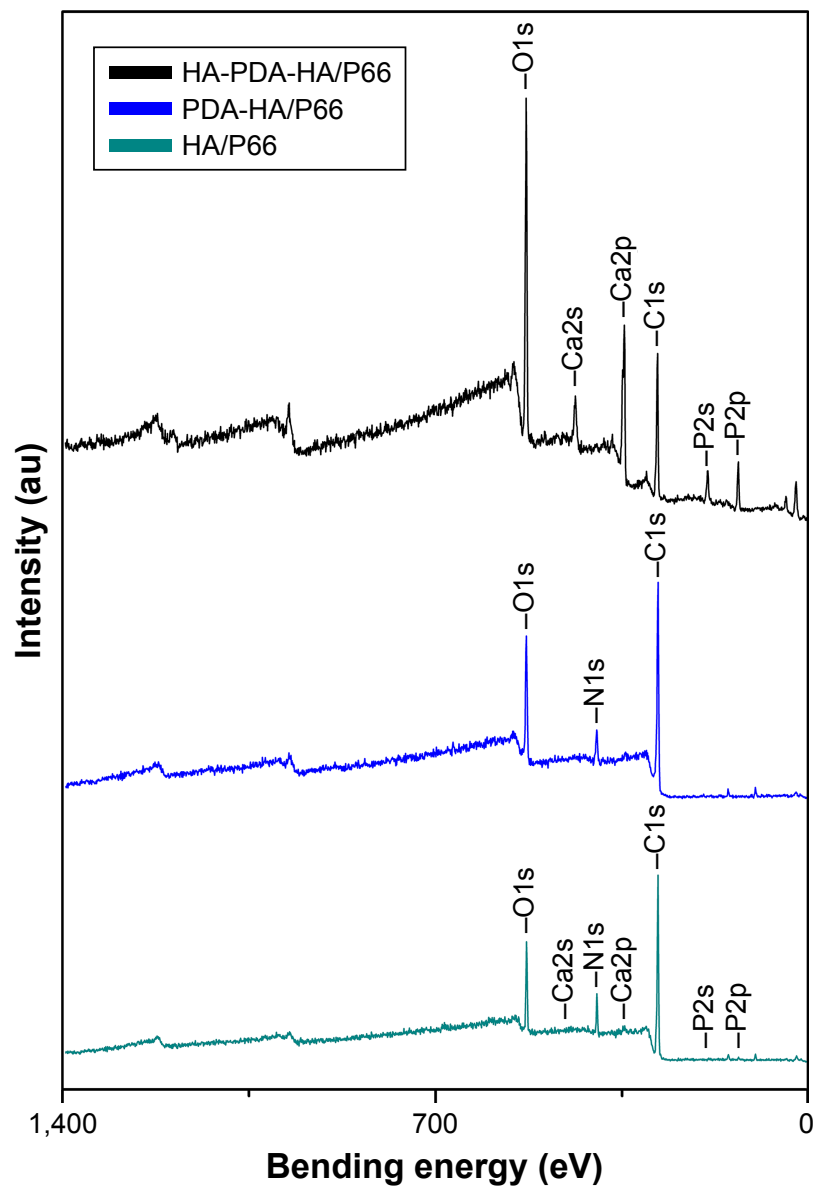

Figure I XPS survey scans identifying surface chemical composition of HA/P66, PDA-HA/P66, and HA-PDA-HA/P66.

Abbreviations: HA/P66, hydroxyapatite/polyamide 66; PDA, polydopamine; XPS, X-ray photoelectron spectroscopy; PDA-HA/P66, polydopamine coating on hydroxyapatite/polyamide 66; HA-PDA-HA/P66, hydroxyapatite coating formation on hydroxyapatite/polyamide 66 assisted by polydopamine.

shown in Figure 1, and the elemental compositions of each layer are listed in Table 1. The HA/P66 substrates exhibited C (1 second), O (1 second), N (1 second), Ca (2p), and $\mathrm{P}(2 \mathrm{p})$ peaks, but the PDA-coated substrates exhibited only C (1 second), O (1 second), and N (1 second) peaks. After soaking in SBF solution, the intensities of the $\mathrm{Ca}(2 \mathrm{p}, 2 \mathrm{~s})$ and $\mathrm{P}(2 \mathrm{p}, 2 \mathrm{~s})$ peaks increased significantly, which indicated the formation of a calcium-phosphate compound on the PDA film. The content of calcium and phosphorus was very low on the surface of HA/P66 but significantly increased on the surface of HA-PDA-HA/P66 (Table 1).

The SEM images of HA/P66 indicated that tiny material debris found on its surface, which were produced by computer numerical control lathe (Figure 2A); the edges of traces and tiny material debris were sharp, whereas, after PDA film formation, their edges became smooth and the material debris became higher and bigger, which resulted in a more uneven surface (Figure 2B). The SEM images of HA-PDA-HA/P66 showed a large number of spherical particles that were 

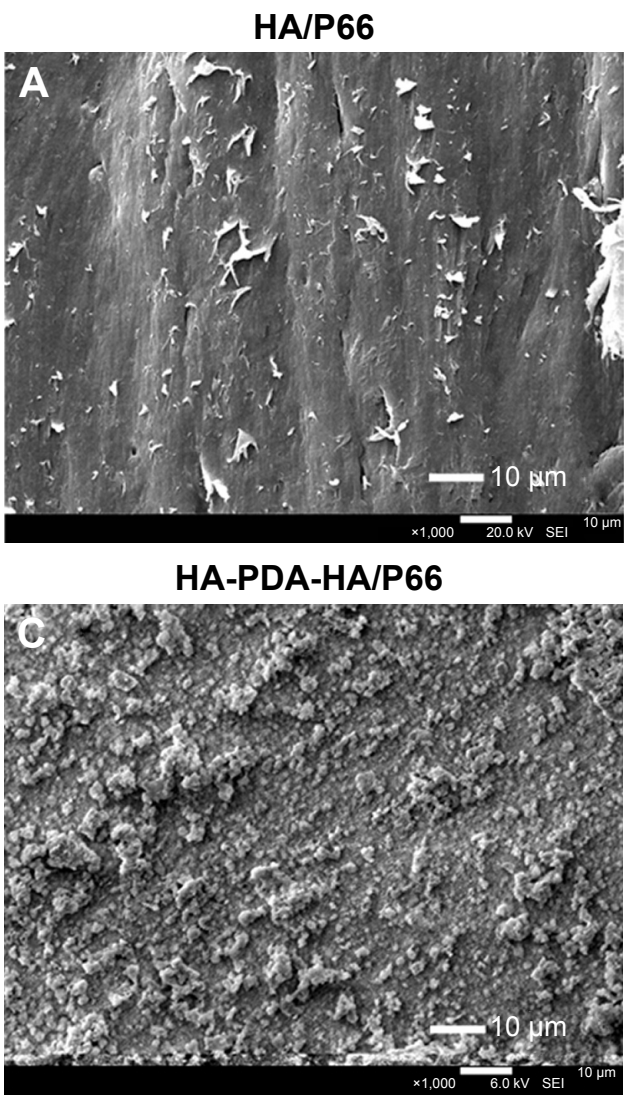

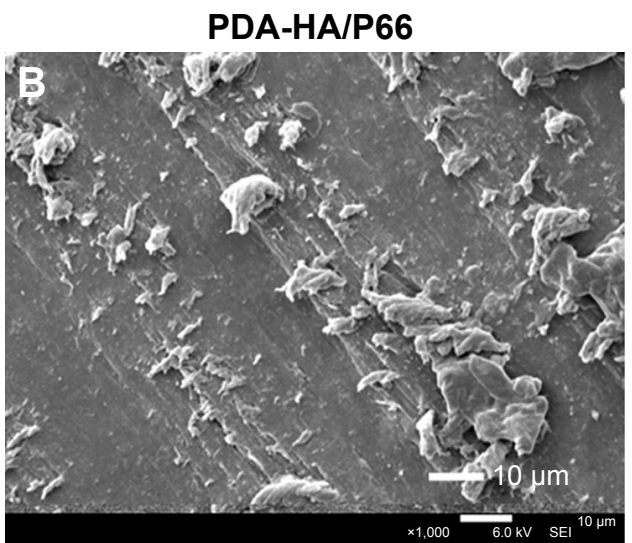

HA-PDA-HA/P66

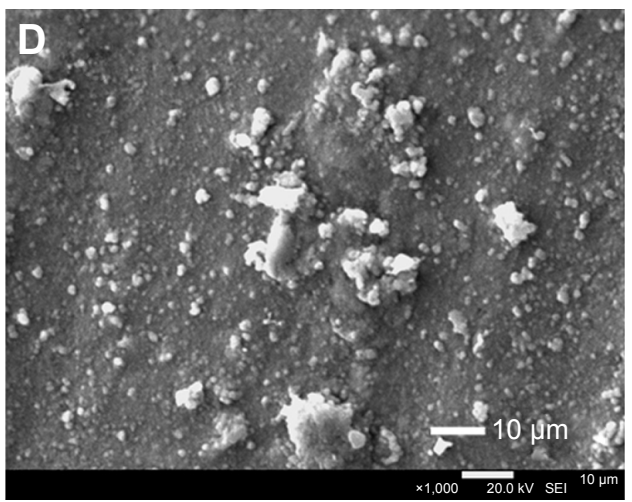

Figure 2 SEM images of HA/P66 (A) and PDA-HA/P66 (B), and SEM images of HA-PDA-HA/P66 before (C) and after (D) exposure to ultrasonication. (Magnification I,000x).

Abbreviations: HA/P66, hydroxyapatite/polyamide 66; PDA, polydopamine; SEM, scanning electron microscopy; PDA-HA/P66, polydopamine coating on hydroxyapatite/ polyamide 66; HA-PDA-HA/P66, hydroxyapatite coating formation on hydroxyapatite/polyamide 66 assisted by polydopamine.

uniformly distributed on the whole surface (Figure 2C). The HA coating remained stable after strong ultrasonication for 1 hour (Figure 2D). The weight of the HA-PDA-HA/P66 substrate was reduced by $21.7 \%$ after sonication, indicating that $78.3 \%$ of the HA was still firmly attached to the underlying PDA-HA/P66 substrate. The XRD image of PDA-HA/ P66 was almost identical to that of HA/P66, and the characteristic P66 peak was observed at $20^{\circ}$ and $23^{\circ}$ in the two XRD images (Figure 3A). In contrast, the characteristic HA peak was observed in the XRD image of HA-PDA-HA/P66, while no characteristic P66 peak was observed, suggesting that the HA coating had formed successfully on the PDA film.

Table I The elemental compositions of unmodified and modified HA/P66 substrates analyzed by XPS and expressed as atomic concentration in percentage

\begin{tabular}{|l|l|l|l|l|l|l|}
\hline Element & $\mathbf{C}$ & $\mathbf{N}$ & $\mathbf{C a}$ & $\mathbf{P}$ & $\mathbf{O}$ & Others \\
\hline HA/P66 & 75.7 & 7.7 & 0.1 & 0.4 & 13.8 & 2.2 \\
\hline PDA-HA/P66 & 70.9 & 8.4 & 0 & 0 & 18.3 & 2.4 \\
\hline HA-PDA-HA/P66 & 35.2 & 0.8 & 11.8 & 10.7 & 41.6 & 0 \\
\hline
\end{tabular}

Abbreviations: HA/P66, hydroxyapatite/polyamide 66; PDA, polydopamine; XPS, X-ray photoelectron spectroscopy; PDA-HA/P66, polydopamine coating on hydroxyapatite/polyamide 66; HA-PDA-HA/P66, hydroxyapatite coating formation on hydroxyapatite/polyamide 66 assisted by polydopamine.
Figure $3 \mathrm{~B}$ and $\mathrm{C}$ depicts the in vitro release profile of both the $\mathrm{Ca}$ and $\mathrm{PO}_{4}^{3-}$ ions during the $\mathrm{HA}$ coating dissolution in 7 days. The $\mathrm{Ca}$ and $\mathrm{PO}_{4}^{3-}$ ions had almost the same release profile, and they were continuously released in a slow manner.

The water contact angles of the HA/P66, PDA-HA/ P66, and HA-PDA-HA/P66 substrates were $70.22^{\circ} \pm 3.67^{\circ}$, $42.98^{\circ} \pm 1.99^{\circ}$, and $20.52^{\circ} \pm 6.79^{\circ}$, respectively (Figure 4 ). The hydrophilicity of PDA-HA/P66 was better $(P<0.05)$ than HA/P66, but worse $(P<0.05)$ than HA-PDA-HA/P66 (Figure 4B). Therefore, it is likely that both PDA and HA coatings could improve the hydrophilicity of the substrate materials. Table 2 summarizes the surface roughness of the HA/P66, PDA-HA/P66, and HA-PDA-HA/P66 substrates. The average roughness $(\mathrm{Ra})$ of the HA/P66, PDA-HA/ P66, and HA-PDA-HA/P66 substrates was 0.41, 0.623, and $0.996 \mu \mathrm{m}$, respectively. Thus, compared with HA/P66, the Ra of PDA-HA/P66 and HA-PDA-HA/P66 substrates increased by $52 \%$ and $143 \%$, respectively.

\section{Cell attachment and proliferation}

The adhesion of the C3H10T1/2 cells on the substrates of the three groups was measured using the CCK-8 assay and 

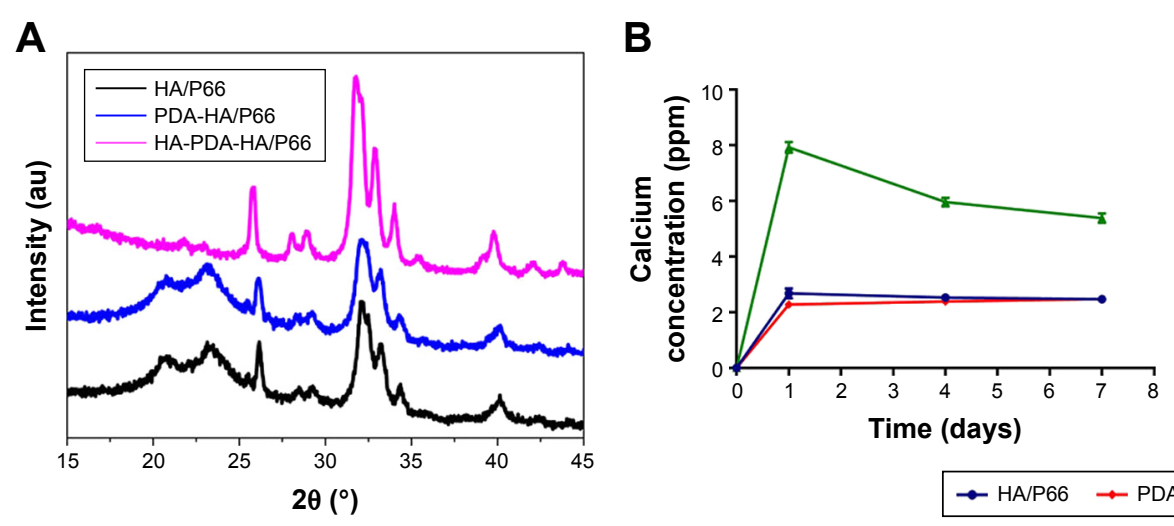

C

Figure 3 XRD profiles of HA/P66, PDA-HA/P66, and HA-PDA-HA/P66 (A). In vitro dissolution and release profiles of calcium and phosphate ions in 7 days (B, C). Abbreviations: HA/P66, hydroxyapatite/polyamide 66; PDA, polydopamine; XRD, X-ray diffraction; PDA-HA/P66, polydopamine coating on hydroxyapatite/polyamide 66; HA-PDA-HA/P66, hydroxyapatite coating formation on hydroxyapatite/polyamide 66 assisted by polydopamine.

is shown in Figure 5. The modified OD value of each group increased with time. After coculturing for 6 and 12 hours, respectively, the OD value of PDA-HA/P66 was obviously higher than that of HA/P66 $(P<0.05)$ but significantly lower than that of HA-PDA-HA/P66 $(P<0.05)$.

The proliferation of the $\mathrm{C} 3 \mathrm{H} 10 \mathrm{~T} 1 / 2$ cells was also measured using the CCK-8 assay after the cells were cocultured with the substrates for 1, 4, and 7 days (Figure 6). The OD values of the HA-PDA-HA/P66 and PDA-HA/P66 substrates obviously increased with time, while the OD values of HA/P66 only increased slightly (Figure 6A). The relative proliferation rates of HA-PDA-HA/P66 and PDA-HA/P66
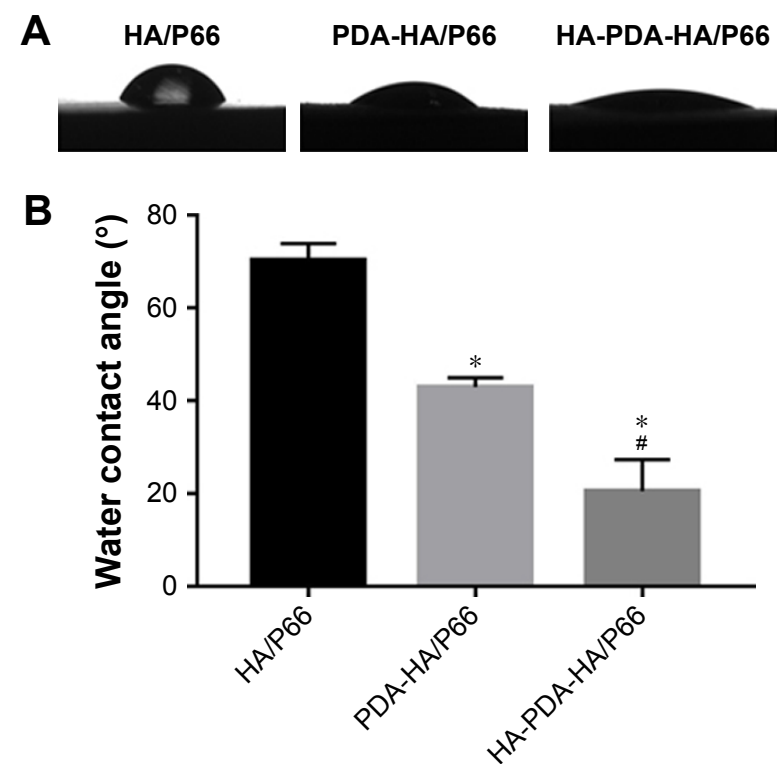

Figure 4 Water contact angles of HA/P66, PDA-HA/P66, and HA-PDA-HA/P66. Notes: (A) The images of water contact angles. (B) Quantitative analysis of water contact angles. *Significant difference compared with HA/P66 $(P<0.05)$. "Significant difference compared with PDA-HA/P66 $(P<0.05)$. Data are presented as mean $\pm S D$. Abbreviations: HA/P66, hydroxyapatite/polyamide 66; PDA, polydopamine; PDAHA/P66, polydopamine coating on hydroxyapatite/polyamide 66; HA-PDA-HA/ P66, hydroxyapatite coating formation on hydroxyapatite/polyamide 66 assisted by polydopamine. were higher $(P<0.05)$ than that of HA/P66 at 4 and 7 days; furthermore, the relative proliferation rate of HA-PDA-HA/ P66 was also higher $(P<0.05)$ than that of PDA-HA/P66 (Figure 6B).

Figure 7 shows the SEM images of the $\mathrm{C} 3 \mathrm{H} 10 \mathrm{~T} 1 / 2$ cells after being cultured on the HA/P66, PDA-HA/P66, and HA-PDA-HA/P66 substrates for 3 and 7 days. Although the number of cells on all samples increased with time, the number of cells on the HA-PDA-HA/P66 sample was obviously greater than that on the PDA-HA/P66 and HA/P66 substrates at 3 days. Furthermore, the cells on the HAPDA-HA/P66 and PDA-HA/P66 substrates overlapped and grew together and covered almost the entire surface at 7 days, while the cells on the HA/P66 substrate grew only along the surface and covered parts of it.

\section{Cell spreading}

The CLSM images revealed that the C3H10T1/2 cells on the HA-PDA-HA/P66 substrate exhibited confluent, multilayer, and clustering morphology. Moreover, the cells were linked to adjacent cells by a greater number of actin filaments. However, the cells on the PDA-HA/P66 and HA/P66 substrates appeared to be in monolayers and were dispersive with fewer actin filaments (Figure 8A).

SEM images showed that the $\mathrm{C} 3 \mathrm{H} 10 \mathrm{~T} 1 / 2$ cells on the HA-PDA-HA/P66 and PDA-HA/P66 substrates spread better

Table 2 Surface roughness of HA/P66, PDA-HA/P66, and HAPDA-HA/P66

\begin{tabular}{|l|l|l|l|}
\hline Sample & HA/P66 & PDA-HA/P66 & HA-PDA-HA/P66 \\
\hline $\mathrm{Ra}(\mu \mathrm{m})$ & $0.410 \pm 0.065$ & $0.623 \pm 0.059 *$ & $0.996 \pm 0.138^{*, \#}$ \\
\hline
\end{tabular}

Notes: ${ }^{*} P<0.05$ compared with HA/P66. ${ }^{*} P<0.05$ compared with PDA-HA/P66. Data are presented as mean \pm SD.

Abbreviations: Ra, average roughness; HA/P66, hydroxyapatite/polyamide 66; PDA, polydopamine; PDA-HA/P66, polydopamine coating on hydroxyapatite/polyamide 66; HA-PDA-HA/P66, hydroxyapatite coating formation on hydroxyapatite/ polyamide 66 assisted by polydopamine. 


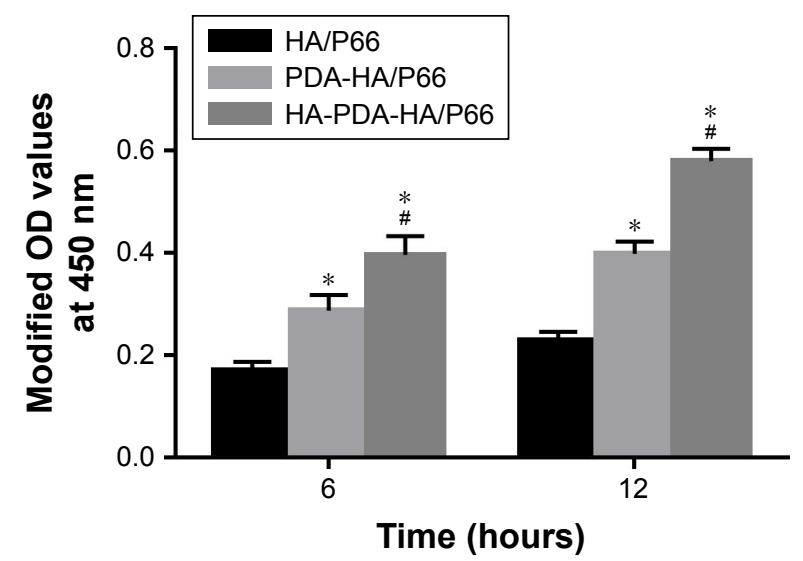

Figure 5 Cell attachment on the substrates assessed by CCK-8 assay.

Notes: *Significant difference compared with HA/P66 ( $P<0.05, n=3)$. \#Significant difference compared with PDA-HA/P66 $(P<0.05, \mathrm{n}=3)$.

Abbreviations: CCK-8, cell counting kit-8; HA/P66, hydroxyapatite/polyamide 66; PDA, polydopamine; PDA-HA/P66, polydopamine coating on hydroxyapatite/ polyamide 66; HA-PDA-HA/P66, hydroxyapatite coating formation on hydroxyapatite/polyamide 66 assisted by polydopamine.

than on the HA/P66 substrate (Figure $8 \mathrm{~B}$ and $\mathrm{C}$ ). In addition, the cells anchored to the surface of HA-PDA-HA/P66 and PDA-HA/P66 seemed more efficient and had a greater number of pseudopods than the cells on the HA/P66 substrates.

\section{ALP activity}

Figure 9 shows the ALP activity of the $\mathrm{C} 3 \mathrm{H} 10 \mathrm{~T} 1 / 2$ cells after the cells were cocultured with the HA/P66, PDA-HA/P66, and HA-PDA-HA/P66 substrates for 7, 10, and 14 days. The ALP activity of the three groups increased with time such that the ALP activity of HA-PDA-HA/P66 and PDA-HA/P66 was higher $(P<0.05)$ than that of HA/P66 at each time point; furthermore, the ALP activity of HA-PDA-HA/P66 was higher $(P<0.05)$ than that of PDA-HA/P66 (Figure 9).
This indicates that both PDA and HA coatings could induce early osteogenesis.

\section{Alizarin red staining and quantitative analysis}

Alizarin red staining was performed after the C3H10T1/2 cells were cocultured in osteogenic induction medium for 21 days (Figure 10A). The staining intensity of HA-PDA-HA/ P66 was stronger than that of PDA-HA/P66 and HA/P66 at 21 days. In addition, the staining intensity of PDA-HA/P66 was stronger than that of HA/P66. The same results were also obtained by quantitative analysis (Figure 10B).

\section{Expression of osteogenic differentiation- related protein}

Figure 11A shows the expression of the osteogenic differentiation-related protein after the $\mathrm{C} 3 \mathrm{H} 10 \mathrm{~T} 1 / 2$ cells were cocultured in osteogenic induction medium for 14,21 , and 28 days. The expression of COL-1, RUNX2, and OCN of the HA-PDA-HA/P66 substrate was much higher $(P<0.05)$ than that of the PDA-HA/P66 and HA/P66 substrates at each time point. The expression of COL-1, RUNX2, and OCN of PDA-HA/P66 was also higher $(P<0.05)$ than that of HA/P66. Figure 11B shows the same results obtained by quantitative analysis.

\section{Micro-CT measurements and histological evaluations}

Micro-CT scanning and histological staining were used to observe bone formation around the implants at 8 weeks after the surgery. The micro-CT two-dimensional (2D) reconstruction images of the longitudinal sections of the bone specimen
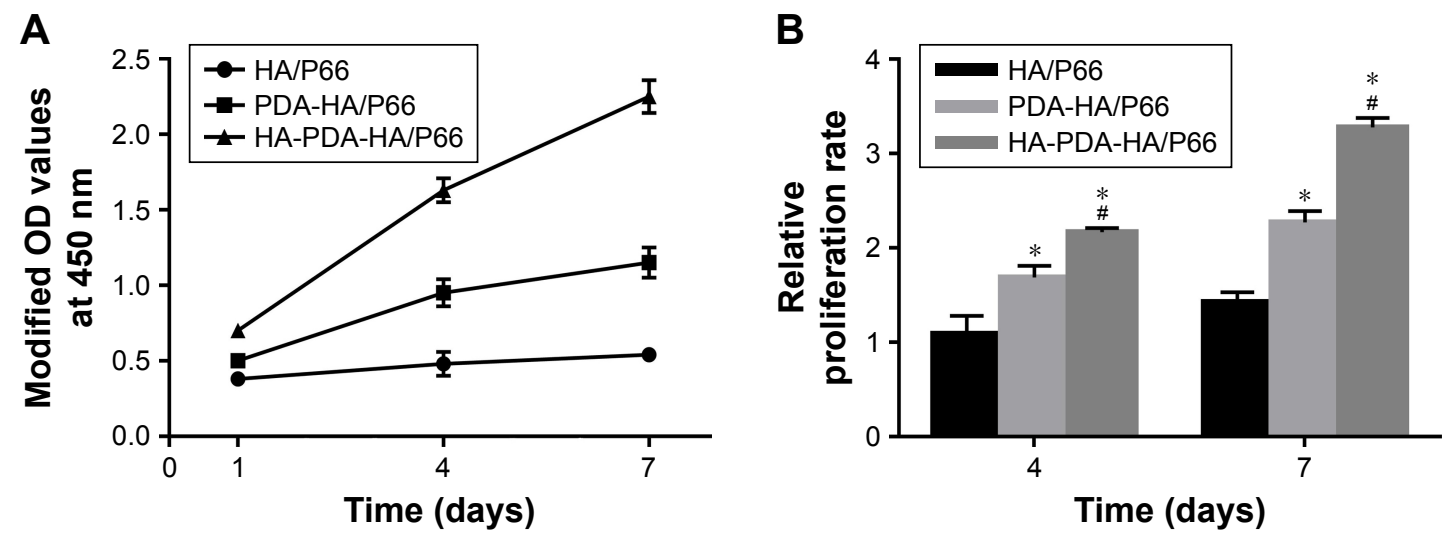

Figure 6 Cell proliferation on the substrates assessed by CCK- 8 assay.

Notes: (A) Proliferative tendency from I to 7 days. (B) Relative proliferation rate. The modified ODs at 4 and 7 days were normalized to the values at I day. $*$ Significant difference compared with HA/P66 $(P<0.05, n=3)$. "Significant difference compared with PDA-HA/P66 $(P<0.05, n=3)$.

Abbreviations: CCK-8, cell counting kit-8; HA/P66, hydroxyapatite/polyamide 66; PDA, polydopamine; PDA-HA/P66, polydopamine coating on hydroxyapatite/polyamide 66; HA-PDA-HA/P66, hydroxyapatite coating formation on hydroxyapatite/polyamide 66 assisted by polydopamine. 

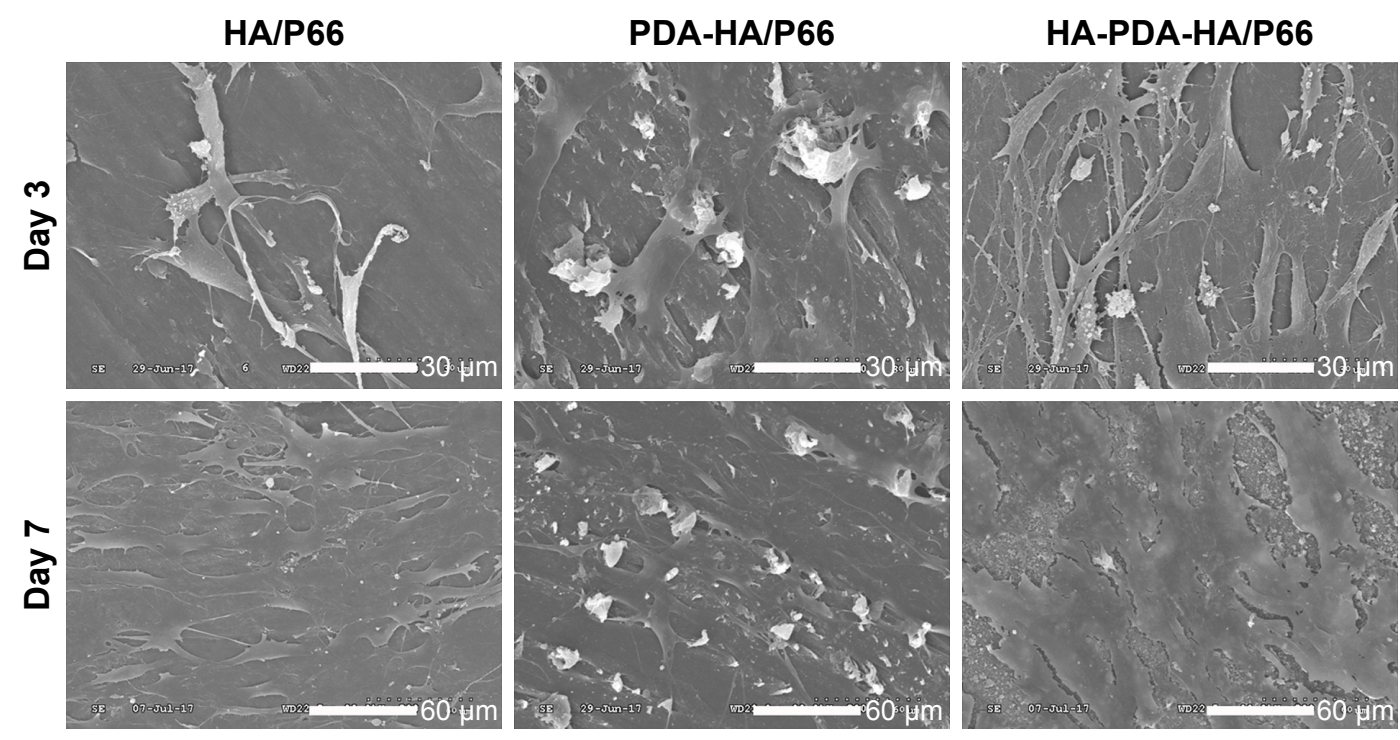

Figure 7 SEM images of the C3HIOTI/2 cells on the substrates at 3 and 7 days (Magnification $600 \times$ for day 3, Magnification 300× for day 7).

Abbreviations: HA/P66, hydroxyapatite/polyamide 66; PDA, polydopamine; SEM, scanning electron microscopy; PDA-HA/P66, polydopamine coating on hydroxyapatite/ polyamide 66; HA-PDA-HA/P66, hydroxyapatite coating formation on hydroxyapatite/polyamide 66 assisted by polydopamine.

as shown in Figure 12A showed the samples' implantation sites and the status of the new bone formation at the interface. As shown in Figure 12A, all samples were basically implanted in the same region of the femoral condyle. One part of the sample was located in the cancellous bone and the other part in the medullary cavity. A larger mass of new bone could be observed at the interface of HA-PDA-HA/P66, while the least amount of new bone was found around HA/P66.
The micro-CT 3D reconstruction images showed that more bone was detected within the VOI around HA-PDA-HA/ P66 than the other two groups (Figure 12B). To quantitatively analyze the formation of new bone, the BV/trabecular volume and the trabecular number of each group were also determined by micro-CT analysis. As shown in Figure 12C and $\mathrm{D}$, the BV/TV and Tb.N of HA-PDA-HA/P66 were significantly higher than those of the PDA-HA/P66 and
A

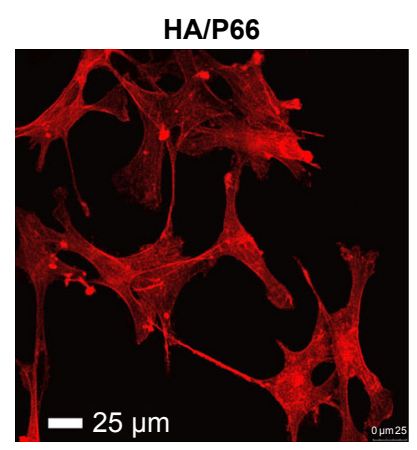

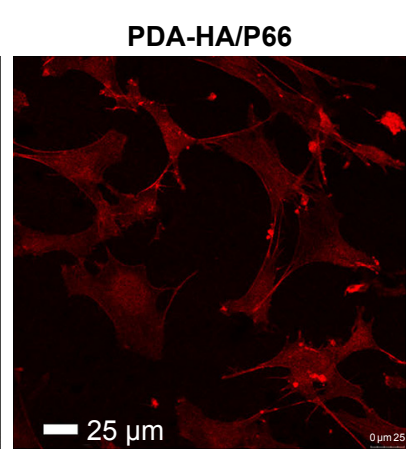

B

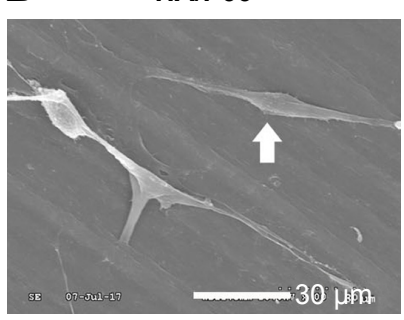

PDA-HA/P66

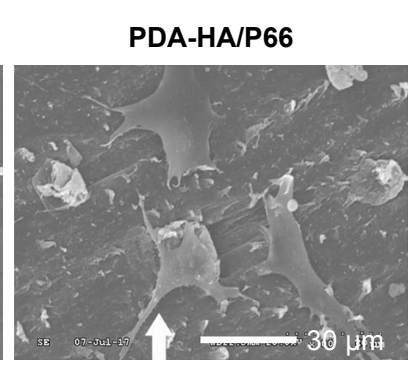

HA-PDA-HA/P66

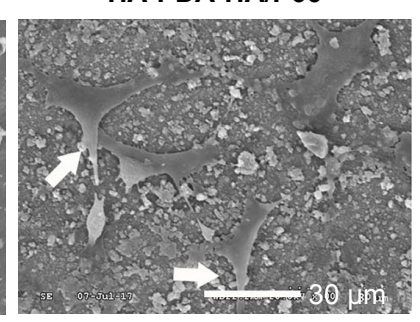

HA-PDA-HA/P66

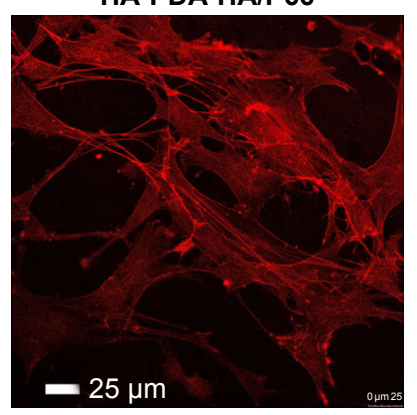

C

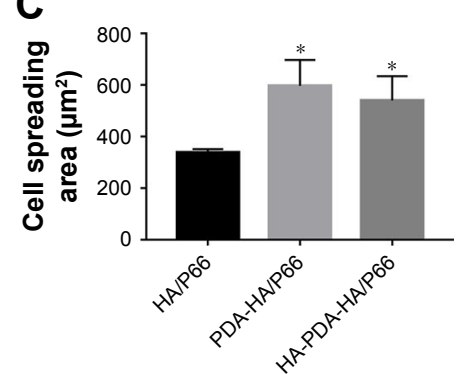

Figure 8 Cell spreading on the substrates at 24 hours, as observed by (A) CLSM and (B) SEM. (C) The cell spreading area was measured with SEM images by Image software. Notes: Arrows indicate cells. *Significant difference compared with HA/P66 $(P<0.05)$.

Abbreviations: CLSM, confocal laser scanning microscopy; HA/P66, hydroxyapatite/polyamide 66; SEM, scanning electron microscopy; PDA-HA/P66, polydopamine coating on hydroxyapatite/polyamide 66; HA-PDA-HA/P66, hydroxyapatite coating formation on hydroxyapatite/polyamide 66 assisted by polydopamine. 


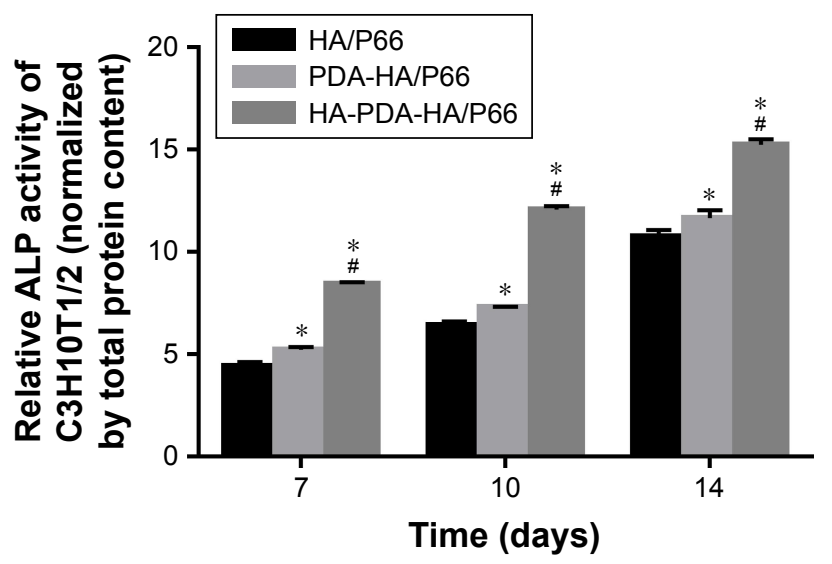

Figure 9 Quantification of ALP activity on the substrates after osteogenic induction for 7,10 , and 14 days.

Notes: ALP activity was normalized to the corresponding total protein content. *Significant difference compared with HA/P66 $(P<0.05)$. "Significant difference compared with PDA-HA/P66 $(P<0.05)$.

Abbreviations: HA/P66, hydroxyapatite/polyamide 66; PDA, polydopamine; PDAHA/P66, polydopamine coating on hydroxyapatite/polyamide 66; HA-PDA-HA/ P66, hydroxyapatite coating formation on hydroxyapatite/polyamide 66 assisted by polydopamine.

HA/P66 groups $(P<0.05)$, while the BV/TV and Tb.N of HA/ P66 were the lowest $(P<0.05)$. Furthermore, similar results were also found using histological staining (Figure 13). The BIC of PDA-HA/P66 was better $(P<0.05)$ than that of HA/P66, but worse $(P<0.05)$ than that of HA-PDA-HA/P66 (Figure 13D). More new bone appeared to form around the HA-PDA-HA/P66 scaffold than the other two scaffolds.

\section{Discussion}

The ideal implant surface modification can achieve faster osseointegration, deliver immediate implant loading, and improve long-term stability. ${ }^{2}$ Osseointegration relies on direct interaction between the bone cells and biomaterial surface. Several studies have shown that surface characterization plays an important role in bone-implant interaction and osseointegration. ${ }^{18,19}$ The preparation of an HA coating is one of the most common methods for surface modification of materials and could improve the process of osseointegration. ${ }^{20}$ In recent times, although several methods were reported for the preparation of the HA coating, only the plasma-spraying coating method was proven clinically useful. However, this method also made it difficult to modify implants with a complex shape. ${ }^{10}$ The plasma-sprayed coatings likely delaminate from the surface of implants because of a dissolution discrepancy between several calcium-phosphate phases in the HA coatings and subsequently result in implant failure. ${ }^{10}$ As the principle of PDA-assisted HA formation is based on the interaction of the catechol groups in PDA with $\mathrm{Ca}-\mathrm{P}$ ions in the SBF solution, the limitations of the plasmasprayed HA coatings are overcome. Hence, we adopted the PDA-assisted biomimetic process to prepare the HA coating on HA/P66.

Many coating parameters can influence the formation of a PDA film, such as coating time, dopamine concentration in the solution, temperature, $\mathrm{pH}$ of Tris- $\mathrm{HCl}$, and oxidation. Researchers have universally agreed that the best dopamine concentration in solution is $2 \mathrm{mg} / \mathrm{mL}$ in Tris-HCL buffer at $\mathrm{pH} 8.5 .^{16,17}$ The PDA coating could attain a thickness of $50 \mathrm{~nm}$ in 24 hours $^{21}$ but could not exceed this limit when the oxidant was oxygen. ${ }^{22}$ Therefore, in our study, we chose to immerse the substrate in dopamine solution $(2 \mathrm{mg} / \mathrm{mL}$,
A
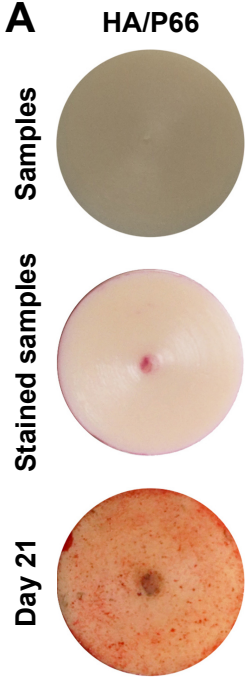

PDA-HA/P66 HA-PDA-HA/P66
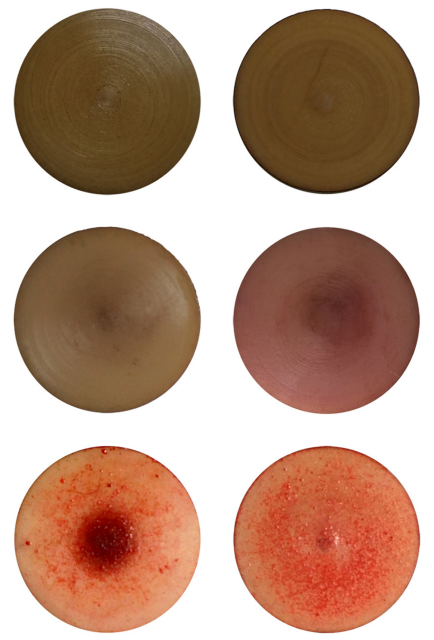

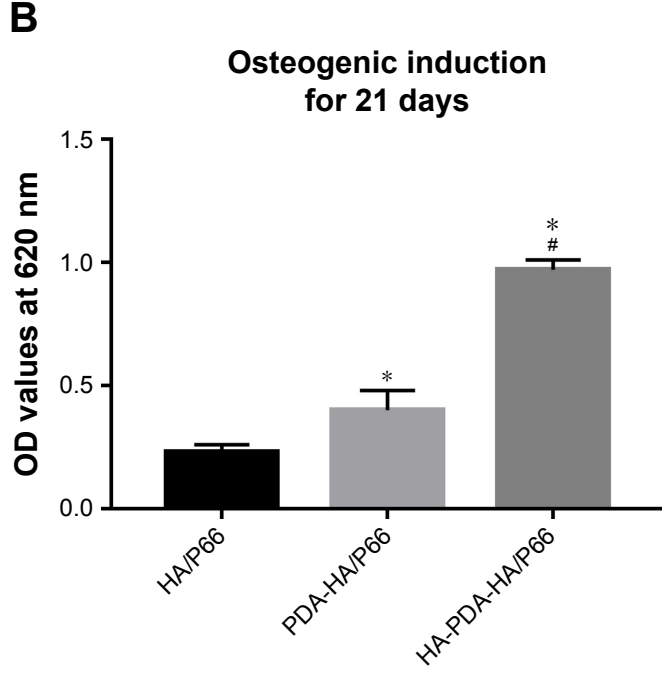

\section{Osteogenic induction for 21 days}

Figure 10 Alizarin red staining and quantitative analysis of mineralization on the substrates.

Notes: (A) Substrates, stained substrates without cells, and stained substrates with cells at 21 days. (B) Colorimetric quantification of the extracellular matrix mineralization at 21 days. *Significant difference compared with HA/P66 $(P<0.05)$. "Significant difference compared with PDA-HA/P66 (P<0.05).

Abbreviations: HA/P66, hydroxyapatite/polyamide 66; PDA, polydopamine; PDA-HA/P66, polydopamine coating on hydroxyapatite/polyamide 66; HA-PDA-HA/P66, hydroxyapatite coating formation on hydroxyapatite/polyamide 66 assisted by polydopamine. 


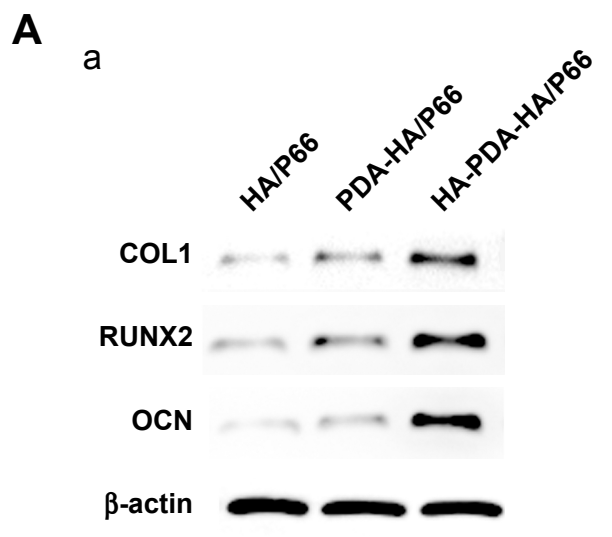

B

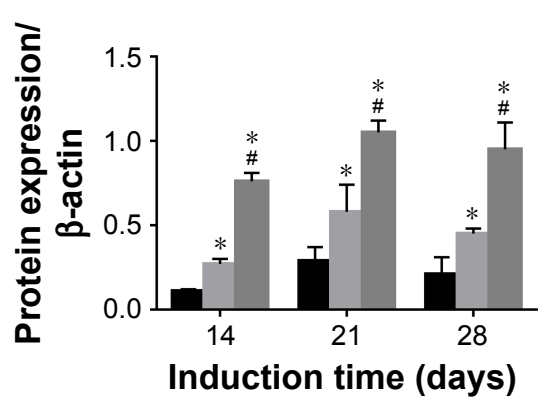

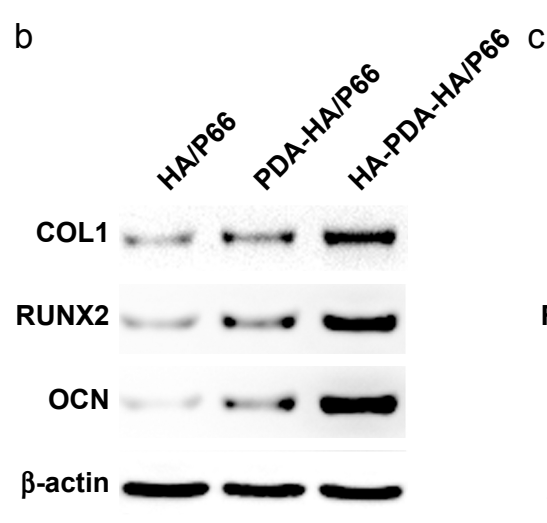

RUNX2

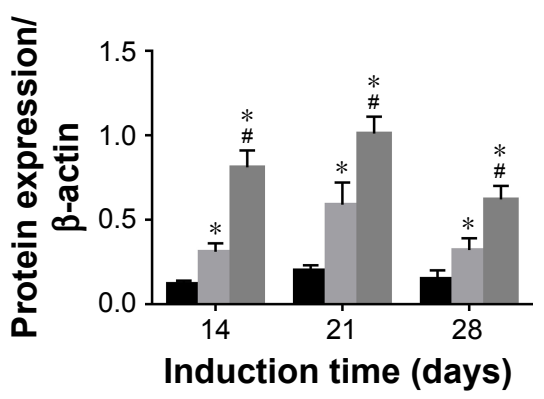

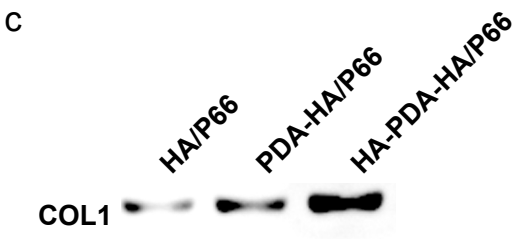

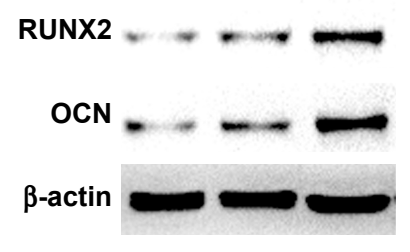

OCN

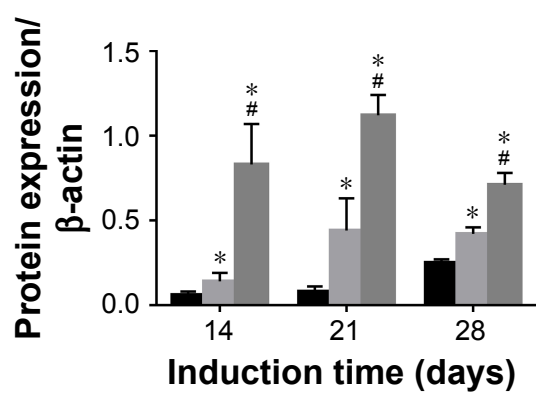

HA/P66 PDA-HA/P66 HA-PDA-HA/P66

Figure II The expression of osteogenic differentiation-related proteins (COLI, RUNX2, and OCN) of C3HI0TI/2 cells cocultured with different substrates in osteogenic inductive medium after (a) 14 days, (b) 21 days, (c) 28 days.

Notes: (A) The images of electrophoretic bands. (B) Quantitative analysis of gray values. *Significant difference compared with HA/P66 ( $P<0.05)$. ${ }^{\#}$ Significant difference compared with PDA-HA/P66 $(P<0.05)$.

Abbreviations: COL I, collagen type I; HA/P66, hydroxyapatite/polyamide 66; OCN, osteocalcin; PDA, polydopamine; RUNX2, runt-related transcription factor 2; PDA-HA/P66, polydopamine coating on hydroxyapatite/polyamide 66; HA-PDA-HA/P66, hydroxyapatite coating formation on hydroxyapatite/polyamide 66 assisted by polydopamine.

pH 8.5) for 24 hours. As shown in Figure 10A, the color of HA/P66 turned dark brown after the substrate was soaked in dopamine solution for 24 hours, indicating catechol oxidation and dopamine polymerization. ${ }^{23}$ As summarized in Table 1, the nitrogen-to-carbon signal (N/C) ratio of the PDA-HA/P66 substrate is 0.12 , similar to the theoretical value of dopamine (N/C 0.125), ${ }^{15}$ which indicates that the coating was derived from dopamine. This is suggestive of the successful coating of the PDA film on the HA/P66 substrate. A previous study showed that preparing PDA on substrates could provide many hydroxyl groups $\left(\mathrm{OH}^{-}\right)$to attract calcium ions $\left(\mathrm{Ca}^{2+}\right)$ and subsequently bond with phosphate ions $\left(\mathrm{PO}_{4}^{3-}\right)$ in the SBF to form HA. ${ }^{16}$ The SEM image initially showed significant numbers of spherical particles formed on the surface of the HA-PDA-HA/P66 substrate. The XPS then confirmed that the spherical particles mainly consisted of calcium and phosphorus, and XRD showed that the XRD pattern of HAPDA-HA/P66 had the characteristic diffraction peak of HA. These results confirmed that the HA coating was successfully prepared on the HA/P66 substrate after sequential soaking in dopamine and 1.5SBF solutions.

Surface characteristics such as surface chemistry, topography, hydrophilicity, and surface roughness obviously affect the biocompatibility of biomaterials. ${ }^{24}$ The XPS results showed that the formation of HA and PDA coatings greatly changed the surface elemental composition of HA/P66. As shown in the SEM images, the topography of PDA-HA/ P66 and HA-PDA-HA/P66 became more uneven than HA/ P66. Our results were consistent with a previous study that reported a concavo-convex surface after the formation of the PDA film. ${ }^{16}$ In this study, the Ra values of HA-PDA-HA/P66 $(0.996 \mu \mathrm{m})$ and PDA-HA/P66 $(0.623 \mu \mathrm{m})$ were significantly greater than that of HA/P66 $(0.41 \mu \mathrm{m})$. These results were in good agreement with the previous results that both the PDA and HA coatings could improve the surface roughness. ${ }^{25,26}$ Furthermore, the water contact angles of HA-PDA-HA/P66 $\left(20.52^{\circ}\right)$ and PDA-HA/P66 (42.98 $)$ were much smaller than that of HA/P66 $\left(70.22^{\circ}\right)$. This suggested that both 
A

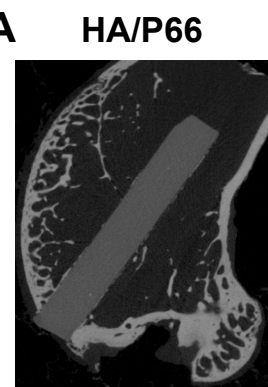

B HA/P66

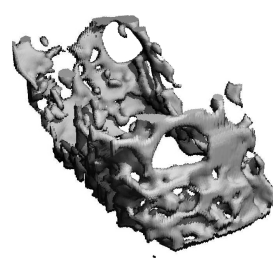

PDA-HA/P66

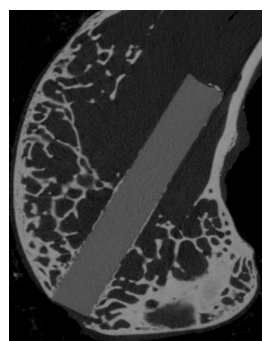

PDA-HA/P66

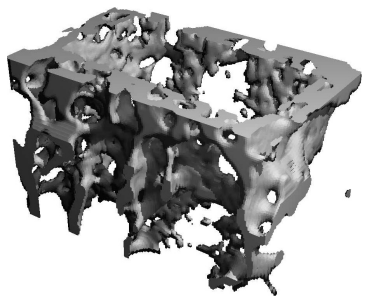

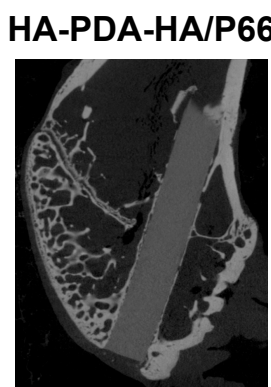

HA-PDA-HA/P66

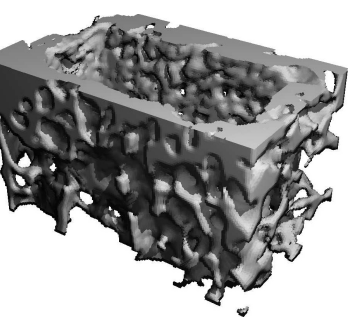

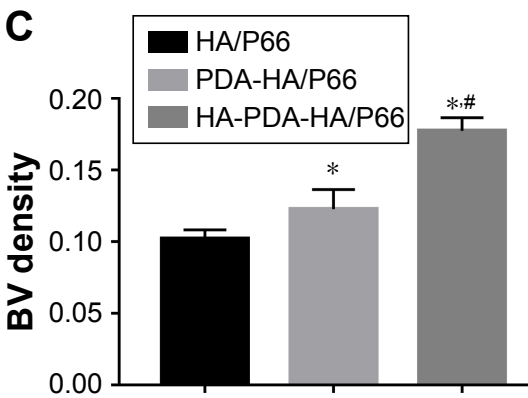

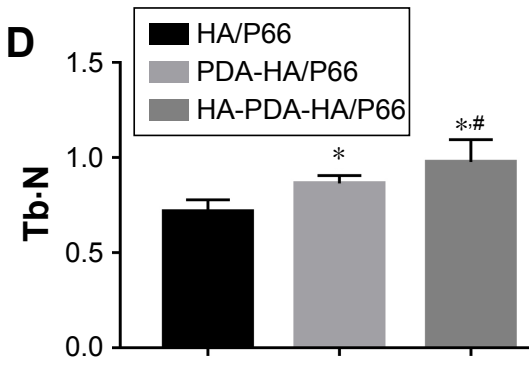

Figure 12 Micro-CT images of femoral condyle defects at 8 weeks after the surgery.

Notes: (A) The longitudinal section images. (B) Micro-CT 3D reconstruction of peri-implant bone with I mm. (C) BV density (BV/TV\%). (D) Tb.N. *Significant difference compared with HA/P66 $(P<0.05)$. "Significant difference compared with PDA-HA/P66 $(P<0.05)$.

Abbreviations: BV, bone volume; HA/P66, hydroxyapatite/polyamide 66; micro-CT, micro-computed tomography; PDA, polydopamine; Tb.N, trabecular number; 3D, three-dimensional; TV, tissue volume; PDA-HA/P66, polydopamine coating on hydroxyapatite/polyamide 66; HA-PDA-HA/P66, hydroxyapatite coating formation on hydroxyapatite/polyamide 66 assisted by polydopamine.
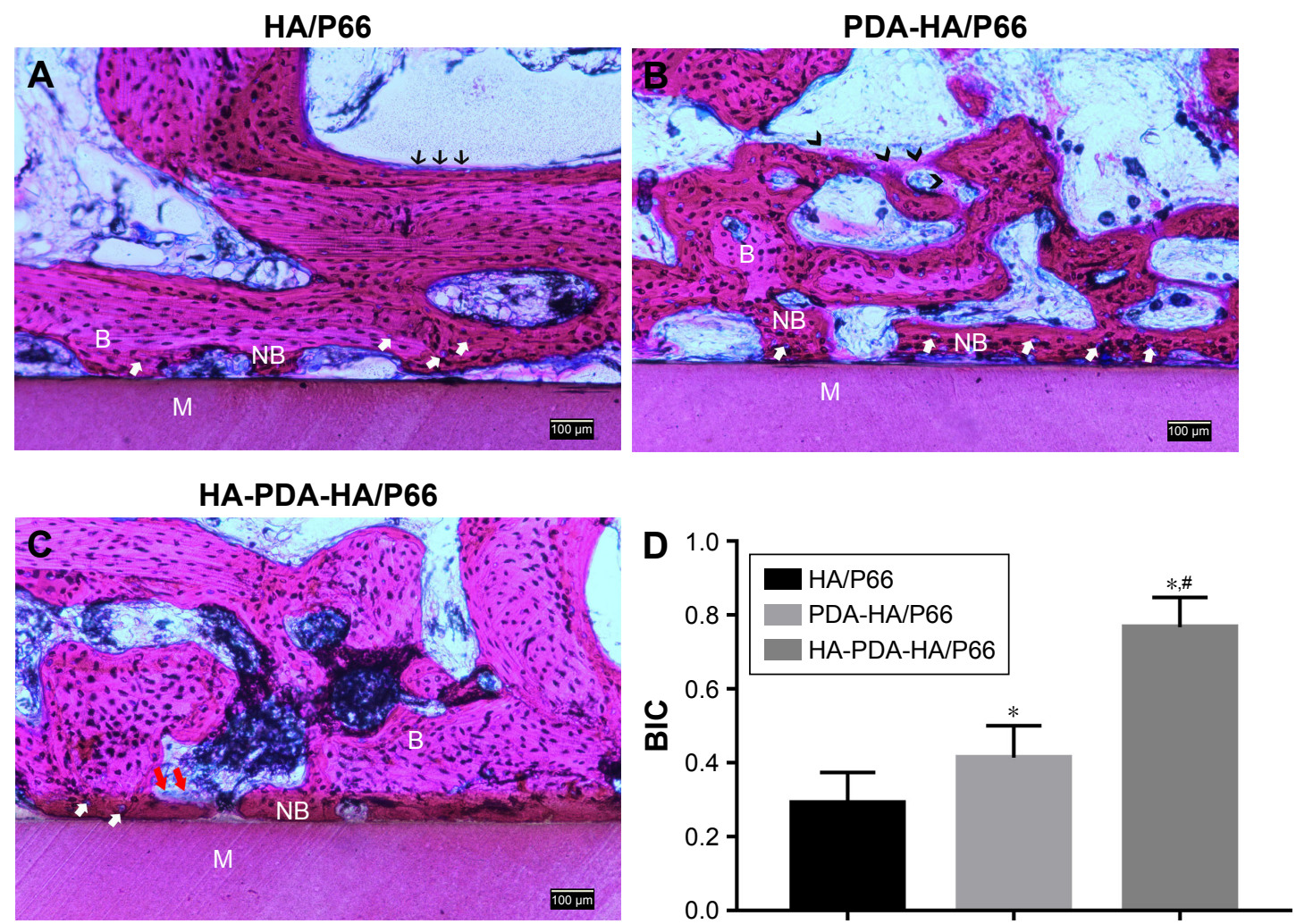

Figure 13 Histological analysis (H\&E staining, $\times 100)$ of femoral condyle defects at 8 weeks after the surgery.

Notes: (A) HA/P66. (B) PDA-HA/P66. (C) HA-PDA-HA/P66. (D) BIC\%. White arrows indicate osteocytes, red arrows indicate osteoclasts-like cells, black arrows indicate osteoblasts, and black tailless arrows indicate osteoid. *Significant difference compared with HA/P66 ( $P<0.05)$. "Significant difference compared with PDA-HA/P66 ( $<<0.05)$. Abbreviations: B, bone; BIC, bone-implant contact; HA/P66, hydroxyapatite/polyamide 66; M, material; NB, new bone; PDA, polydopamine; PDA-HA/P66, polydopamine coating on hydroxyapatite/polyamide 66; HA-PDA-HA/P66, hydroxyapatite coating formation on hydroxyapatite/polyamide 66 assisted by polydopamine. 
PDA and HA coatings improved the hydrophilicity of the HA/P66 substrate. These results indicated that the formation of PDA and HA coatings on the HA/P66 substrate changed not only the surface chemistry and topography but also the hydrophilicity and surface roughness. A previous study has shown that the bonding strength of coatings with substrates and the degradation rate of coatings are two important factors in maintaining the longevity of implants. ${ }^{27}$ In this study, most HA was still firmly attached to the underlying PDA-HA/P66 substrate despite strong ultrasound treatment for 1 hour. The release test showed that $\mathrm{Ca}$ and $\mathrm{PO}_{4}^{3-}$ ions were continuously released in a controlled manner. These properties of the HA coating increase its potential in clinical applications, as they improve the life span of implants.

Early cell adhesion and spreading is essential for subsequent cell growth, proliferation, and differentiation. ${ }^{28}$ In this study, a greater number of $\mathrm{C} 3 \mathrm{H} 10 \mathrm{~T} 1 / 2$ cells grew on the HA-PDA-HA/P66 substrate with more pseudopods and actin filaments than on the HA/P66. The CCK-8 assay results and SEM analysis showed that as compared with the HA/P66 substrate both PDA-HA/P66 and HA-PDA-HA/ P66 could facilitate the proliferation of $\mathrm{C} 3 \mathrm{H} 10 \mathrm{~T} 1 / 2$ cells with HA-PDA-HA/P66 faring better than PDA-HA/P66. These results indicated that HA-PDA-HA/P66 obviously facilitated cell attachment, spreading, and proliferation. It has been found that improvement in surface hydrophilicity could promote cell attachment and spreading. ${ }^{29,30}$ Surface roughness increased cell attachment owing to its increased protein absorption. ${ }^{31}$ In addition, research has shown that HA has good biocompatibility and bioactivity, ${ }^{32}$ and PDA improves the bioactive behavior of materials owing to its catecholamine structure. ${ }^{33}$ Therefore, it was suggested that the mechanism of positive response of $\mathrm{C} 3 \mathrm{H} 10 \mathrm{~T} 1 / 2$ cells to the HA and PDA coatings could be attributed to the change in the surface chemistry and improvement in hydrophilicity and surface roughness.

Osteogenic differentiation of cells around the boneimplant interface affects bone remodeling. It is known that ALP is a sign of early osteogenic differentiation, while the formation of calcium nodules is a sign of late osteogenic differentiation. Higher levels of ALP activity indicated that cells cultured on the materials differentiated more rapidly and earlier. In this study, the C3H10T1/2 cells on the HAPDA-HA/P66 substrate showed a higher ALP activity and formed more calcium nodules than those on PDA-HA/P66, whereas the cells on HA/P66 showed the lowest ALP activity and formed the fewest calcium nodules. These results proved that, while both HA and PDA coatings could facilitate osteogenic differentiation of C3H10T1/2 cells, HA fared better than PDA. To further study the osteogenic ability of different substrates, the expression levels of osteogenic differentiation-related proteins (COL1, RUNX2, and OCN) were determined by Western blotting. Synthesis of osteogenic differentiation-related proteins such as COL1, RUNX2, and OCN reflects early, intermediate, and late osteogenic differentiation, respectively. ${ }^{34}$ At each time point, the $\mathrm{C} 3 \mathrm{H} 10 \mathrm{~T} 1 / 2$ cells on the HA-PDA-HA/P66 substrate showed significantly higher expression of COL1, RUNX2, and OCN than those on PDA-HA/P66, while the cells on HA/P66 showed lower expression than the other two groups. These results further confirmed that HA and PDA coatings could promote osteogenic differentiation. These findings are consistent with other studies. ${ }^{35,36}$ A previous study suggested that surface roughness at the micro- and submicro-levels facilitates osteoblast differentiation. ${ }^{37}$ Therefore, the bioactive benefits of the HA coating and surface roughness may be the reasons for the HAPDA-HA/P66 substrate promoting osteogenic differentiation of $\mathrm{C} 3 \mathrm{H} 10 \mathrm{~T} 1 / 2$ cells.

Direct bone attachment and integration at the implant interface plays a critical role in the performance of an implant. The effects of HA and PDA coatings on osseointegration were further studied in vivo. In this study, micro-CT and histological examinations were conducted to detect new bone formation at the bone-implant interface. We observed that newly formed bone was tightly bound to the surface of the HA-PDA-HA/P66 implant. Furthermore, more newly formed bone could be detected at the bone-implant interface of HA-PDA-HA/P66 compared with PDA-HA/P66 and HA/P66. Quantitative analysis of micro-CT further confirmed that more new bone was formed around HA-PDA-HA/P66 than the other two substrates. Histological examination resulted in the same conclusion. Our in vivo and in vitro experimental results showed that HA-PDA-HA/P66 had a more positive effect on osseointegration in vivo than the other two substrates. The reconstruction of bone in vivo is closely correlated with the adhesion, proliferation, and differentiation of osteoblasts in vitro. The reason that the HA coating promotes osteogenesis is likely a combination of increased surface roughness, hydrophilic improvement, and exposure of more bioactive HA particles. These features may provide more osteoconductive and osteoinductive scaffolds for protein binding, which will promote osteoblast adhesion, proliferation, and differentiation. Therefore, a better cellular response caused by the HA coating is likely to produce a greater amount of bone tissue around the HA-PDA-HA/ P66 substrate. 


\section{Conclusion}

In this study, an HA coating was successfully prepared on the surface of the HA/P66 substrate using a PDA-assisted biomimetic process. The formation of the HA coating not only changed the surface chemistry and topography but also improved the hydrophilicity and roughness of the surface material, thereby facilitating adhesion, proliferation, and osteogenic differentiation of the C3H10T1/2 cells. Furthermore, in vivo experiments, micro-CT analysis, and histological findings revealed that the HA coating significantly improved the osteogenic induction of HA/P66. In conclusion, PDA-assisted biomimetic preparation of the HA coating improved the osteogenic inducing ability of the biomaterials and showed potential for the surface modification of implanted materials.

\section{Acknowledgments}

This study was supported by the National Natural Science Foundation for Young Scientists of China (no: 81501876), the Science and Technology Project of Chongqing Education Committee (no: KJ1702031), and the Achievement Transformation Project of Chongqing Education Committee (no: KJZH17110). The authors would like to thank Lihua Peng for his help in cell experiments.

\section{Disclosure}

The authors report no conflicts of interest in this work.

\section{References}

1. Wennerberg A, Albrektsson T. Suggested guidelines for the topographic evaluation of implant surfaces. Int J Oral Maxillofac Implants. 2000;15(3):331-344.

2. Gupta S, Dahiya V, Shukla P. Surface topography of dental implants: a review. J Dent Implants. 2014;4(1):66-71.

3. Puleo DA, Nanci A. Understanding and controlling the bone-implant interface. Biomaterials. 1999;20(23-24):2311-2321.

4. Zhang Y, Deng X, Jiang D, et al. Long-term results of anterior cervical corpectomy and fusion with nano-hydroxyapatite/polyamide 66 strut for cervical spondylotic myelopathy. Sci Rep. 2016;6(1):1-11.

5. Deng QX, Ou YS, Zhu Y, et al. Clinical outcomes of two types of cages used in transforaminal lumbar interbody fusion for the treatment of degenerative lumbar diseases: n-HA/PA66 cages versus PEEK cages. J Mater Sci Mater Med. 2016;27(6):1-9.

6. Cheng C, XiL, WeiH, Ning H, Shijie Y, Mingming Y. Nano-hydroxyapatite/ polyamide 66 biocomposites combined with vascular endothelial growth factor for treating early avascular necrosis of femoral head. China Pharmaceuticals. 2011;27(9):852-857.

7. Qiao B, Li J, Zhu Q, et al. Bone plate composed of a ternary nanohydroxyapatite/polyamide 66/glass fiber composite: biomechanical properties and biocompatibility. Int J Nanomedicine. 2014;9:1423-1432.

8. Zhang S, Yang Q, Zhao W, et al. In vitro and in vivo biocompatibility and osteogenesis of graphene-reinforced nanohydroxyapatite polyamide 66 ternary biocomposite as orthopedic implant material. Int J Nanomedicine. 2016;11:3179-3189.
9. Wang X, Ju F, Li A, et al. Nell-1 Gene Modified Mesenchymal Stem Cells on Biomimetic Porous Nano-Hydroxyapatite/Polyamide 66 Scaffolds Effectively Prevent Nonunion in Rats. J Biomater Tissue Eng. 2016; 6(5):408-416.

10. Le Guéhennec L, Soueidan A, Layrolle P, Amouriq Y. Surface treatments of titanium dental implants for rapid osseointegration. Dent Mater. 2007;23(7):844-854

11. Aruna ST, Kulkarni S, Chakraborty M, Kumar SS, Balaji N, Mandal C. A comparative study on the synthesis and properties of suspension and solution precursor plasma sprayed hydroxyapatite coatings. Ceram Int. 2017;43(13):9715-9722.

12. Sidane D, Rammal H, Beljebbar A, et al. Biocompatibility of sol-gel hydroxyapatite-titania composite and bilayer coatings. Mater Sci Eng C Mater Biol Appl. 2017;72:650-658.

13. Hasan AF, Elttayef AHK, Khalaf MK. The effect of different thermal treatments on corrosion behavior of the hydroxyapatite coated on ti-6al-4v alloy by electrophoretic deposition and dip coating. Ibn AL-Haitham J Pure Appl Sci. 2017;30(1):355-365.

14. Kaynak Bayrak G, Demirtaş TT, Gümüşderelioğlu M. Microwaveinduced biomimetic approach for hydroxyapatite coatings of chitosan scaffolds. Carbohydr Polym. 2017;157:803-813.

15. Lee H, Dellatore SM, Miller WM, Messersmith PB. Mussel-inspired surface chemistry for multifunctional coatings. Science. 2007;318(5849): 426-430.

16. Zhe W, Dong C, Sefei Y, Dawei Z, Kui X, Xiaogang L. Facile incorporation of hydroxyapatite onto an anodized Ti surface via a mussel inspired polydopamine coating. Appl Surf Sci. 2016;378:496-503.

17. Ryu J, Ku SH, Lee H, Park CB. Mussel-Inspired polydopamine coating as a universal route to hydroxyapatite crystallization. Adv Funct Mater. 2010;20(13):2132-2139.

18. Thomsen P, Malmström J, Emanuelsson L, René M, Snis A. Electron beam-melted, free-form-fabricated titanium alloy implants: Material surface characterization and early bone response in rabbits. $J$ Biomed Mater Res B Appl Biomater. 2009;90(1):35-44.

19. Karazisis D, Petronis S, Agheli H, et al. The influence of controlled surface nanotopography on the early biological events of osseointegration. Acta Biomater. 2017;53:559-571.

20. Fouda M, Nemat A, Gawish A, Baiuomy AR. Does the coating of titanium implants by hydroxyapatite affect the elaboration of free radicals. An experimental study. Aust J Basic Appl Sci. 2009;3(2):1122-1129.

21. Zhu B, Edmondson S. Polydopamine-melanin initiators for Surfaceinitiated ATRP. Polymer. 2011;52(10):2141-2149.

22. Müller M, Kessler B. Deposition from dopamine solutions at Ge substrates: an in situ ATR-FTIR study. Langmuir. 2011;27(20):12499-12505.

23. Madhurakkat Perikamana SK, Lee J, Lee YB, et al. Materials from mussel-inspired chemistry for cell and tissue engineering applications. Biomacromolecules. 2015;16(9):2541-2555.

24. Ma T, Ge X, Zhang Y, Lin Y. Effect of titanium surface modifications of dental implants on rapid osseointegration. In: Suzuki KSO, Takahashi N, editors. Oral Health Science 2016 Innovative Research on BiosisAbiosis Intelligent Interface. Singapore: Springer; 2016:247-256.

25. Poh CK, Shi Z, Tan XW, et al. Cobalt chromium alloy with immobilized BMP peptide for enhanced bone growth. J Orthop Res. 2011; 29(9):1424-1430.

26. Chien CY, Liu TY, Kuo WH, Wang MJ, Tsai WB. Dopamine-assisted immobilization of hydroxyapatite nanoparticles and RGD peptides to improve the osteoconductivity of titanium. J Biomed Mater Res A. 2013; 101(3):740-747.

27. Wu C, Chen Z, Yi D, Chang J, Xiao Y, Chengtie Wu Z. Multidirectional effects of Sr-, Mg-, and Si-containing bioceramic coatings with high bonding strength on inflammation, osteoclastogenesis, and osteogenesis. ACS Appl Mater Interfaces. 2014;6(6):4264-4276.

28. Györgyey Á, Ungvári K, Kecskeméti G, et al. Attachment and proliferation of human osteoblast-like cells (MG-63) on laser-ablated titanium implant material. Mater Sci Eng C Mater Biol Appl. 2013;33(7): $4251-4259$. 
29. Zhao MH, Chen XP, Wang Q. Wetting failure of hydrophilic surfaces promoted by surface roughness. Sci Rep. 2014;4:5376.

30. Bruining MJ, Pijpers AP, Kingshott P, Koole LH. Studies on new polymeric biomaterials with tunable hydrophilicity, and their possible utility in corneal repair surgery. Biomaterials. 2002;23(4):1213-1219.

31. Al Qahtani WM, Schille C, Spintzyk S, et al. Effect of surface modification of zirconia on cell adhesion, metabolic activity and proliferation of human osteoblasts. Biomed Tech. 2017;62(1):75-87.

32. Koh KS, Choi JW, Park EJ, Oh TS. Bone regeneration using silk hydroxyapatite hybrid composite in a rat alveolar defect model. Int $J$ Med Sci. 2018;15(1):59-68.

33. Yan P, Wang J, Wang L, Liu B, Lei Z, Yang S. The in vitro biomineralization and cytocompatibility of polydopamine coated carbon nanotubes. Appl Surf Sci. 2011;257(11):4849-4855.
34. Cai Y, Wang X, Poh CK, et al. Accelerated bone growth in vitro by the conjugation of BMP2 peptide with hydroxyapatite on titanium alloy. Colloids Surf B Biointerfaces. 2014;116:681-686.

35. Kao CT, Lin CC, Chen YW, Yeh CH, Fang HY, Shie MY. Poly(dopamine) coating of 3D printed poly(lactic acid) scaffolds for bone tissue engineering. Mater Sci Eng C Mater Biol Appl. 2015;56:165-173.

36. Ko E, Lee JS, Kim H, et al. Electrospun silk fibroin nanofibrous scaffolds with two-stage hydroxyapatite functionalization for enhancing the osteogenic differentiation of human adipose-derived mesenchymal stem cells. ACS Appl Mater Interfaces. 2018;10(9):7614-7625.

37. Gittens RA, McLachlan T, Olivares-Navarrete R, et al. The effects of combined micron-/submicron-scale surface roughness and nanoscale features on cell proliferation and differentiation. Biomaterials. 2011; 32(13):3395-3403.
International Journal of Nanomedicine

\section{Publish your work in this journal}

The International Journal of Nanomedicine is an international, peerreviewed journal focusing on the application of nanotechnology in diagnostics, therapeutics, and drug delivery systems throughout the biomedical field. This journal is indexed on PubMed Central, MedLine, CAS, SciSearch ${ }^{\circledR}$, Current Contents ${ }^{\circledR} /$ Clinical Medicine,

\section{Dovepress}

Journal Citation Reports/Science Edition, EMBase, Scopus and the Elsevier Bibliographic databases. The manuscript management system is completely online and includes a very quick and fair peer-review system, which is all easy to use. Visit http://www.dovepress.com/ testimonials.php to read real quotes from published authors.

Submit your manuscript here: http://www.dovepress.com/international-journal-of-nanomedicine-journal 